\title{
Factors influencing antimicrobial prescribing by Dutch companion animal veterinarians: A qualitative study
}

\author{
Nonke E.M. Hopman ${ }^{\mathrm{a}}$, Marlies E.J.L. Hulscher ${ }^{\mathrm{b}}$, Haitske Graveland ${ }^{\mathrm{a}}$, David C. Speksnijder ${ }^{\mathrm{a}}$, \\ Jaap A. Wagenaar ${ }^{\mathrm{a}, \mathrm{c}}$, Els M. Broens ${ }^{\mathrm{a}, *}$ \\ ${ }^{a}$ Department of Infectious Diseases and Immunology, Faculty of Veterinary Medicine, Utrecht University, Yalelaan 1, 3584 CL Utrecht, The Netherlands \\ ${ }^{\mathrm{b}}$ Scientific Center for Quality of Healthcare (IQ healthcare), Radboud Institute for Health Sciences, Radboud University Medical Center, Geert Grooteplein 21, 6525 EZ \\ Nijmegen, The Netherlands \\ ${ }^{c}$ Wageningen Bioveterinary Research, Houtribweg 39, 8221 RA Lelystad, The Netherlands
}

\section{A R T I C L E I N F O}

\section{Keywords:}

Antimicrobial use

Companion animals

Qualitative research

Prescribing behaviour

Veterinary

\begin{abstract}
A B S T R A C T
Use of antimicrobials selects for antimicrobial resistance, and this poses a threat for both human and animal health. Although previous studies show that total antimicrobial use in Dutch companion animal clinics is relatively low and decreasing, the majority of antimicrobials prescribed are categorised as critically important for human medicine by the World Health Organization (WHO). Large differences in use between clinics are also observed. Identification of factors that influence the prescribing behaviour of veterinarians is needed to tailor future interventions aimed at promoting prudent use of antimicrobials in companion animals. The aim of this study was to explore factors influencing the antimicrobial prescribing behaviour of companion animal veterinarians in the Netherlands.

Face-to-face, semi-structured interviews were used to interview 18 Dutch companion animal veterinarians. Interviews were held until theoretical data saturation was reached. An interview guide was used to structure the interviews, and ATLAS.ti 7.5 was used to manage and analyse the qualitative data. An iterative approach was applied to develop a conceptual model of factors that influence antimicrobial prescribing behaviour.

The conceptual model shows four major categories of factors that influence the antimicrobial prescribing behaviour: veterinarian-related factors, patient-related (i.e. owner- and pet-related) factors, treatment-related factors (i.e. alternative treatment options and antimicrobial-related factors) and contextual factors (i.e. professional interactions, further diagnostics and environmental factors). All four major categories of influencing factors should be addressed to improve awareness on antimicrobial prescribing behaviour and to develop an antimicrobial stewardship programme for companion animal clinics.
\end{abstract}

\section{Introduction}

The global increase in antimicrobial resistance (AMR) is recognised as a threat for both human and animal health (Guardabassi et al., 2004; WHO, 2015; Pomba et al., 2017). As any use of antimicrobials selects for antimicrobial resistance, prudent use of antimicrobials (AMs) in humans and animals should be promoted (Collignon et al., 2016). To support prudent antimicrobial use (AMU) the World Health Organization (WHO) compiled a list of AMs that are critically important for human medicine, the so-called CIA list (WHO, 2017). Recommendations on restricted AMU focus primarily on AMU in food animals, but due to the close contact between companion animals and their owners and therefore the potential transmission of resistant bacteria, these recommendations should likewise hold for companion animals (Guardabassi et al., 2004; Chomel and Sun, 2011; EMA, 2015; Baede et al., 2015; Duim et al., 2016; Marques et al., 2018; Worthing et al., 2018).

In comparison with food animals, data on AMU in companion animals are scarce. Studies in the UK and New Zealand showed that the majority of AMs used in dogs and cats were classified as critically important (CIA) to human health (Pleydell et al., 2012; Buckland et al., 2016). A survey on purchase data from 100 Dutch veterinary clinics providing care for companion animals (2012-2014) showed that the majority of AMs were on the CIA list, while the highest priority AMs

\footnotetext{
* Corresponding author.

E-mail addresses: n.e.m.hopman@uu.nl (N.E.M. Hopman), marlies.hulscher@radboudumc.nl (M.E.J.L. Hulscher), h.graveland@uu.nl (H. Graveland), d.c.speksnijder@uu.nl (D.C. Speksnijder), j.wagenaar@uu.nl (J.A. Wagenaar), e.m.broens@uu.nl (E.M. Broens).
} 
(i.e. $3^{\text {rd }}$ generation cephalosporins and fluoroquinolones) accounted for $7 \%$ of total AMU in 2014. Total AMU was relatively low (compared to AMU in livestock) and a decrease was seen over the study period. Large differences between clinics were observed (e.g. a 13-fold difference in total use between clinics in 2014), indicating that there is room for improvement (Netherlands Veterinary Medicines Institute, 2017).

Several international and national guidelines to promote prudent AMU in companion animals have been developed in recent years (AMCRA; BSAVA; DSAVA (Jessen et al., 2012); ISCAID; WVAB), but the implementation of these guidelines and antimicrobial stewardship programmes in companion animals are limited. Over the past few decades, antimicrobial stewardship programmes have been implemented in human medicine to optimise AMU (Dyar et al., 2017). Before implementing a stewardship programme, it is necessary to know which factors influence antimicrobial prescribing behaviour. In human medicine, these determinants have been studied extensively in different clinical settings (Hulscher et al., 2010a; Teixeira Rodrigues et al., 2013; van Buul et al., 2014). In veterinary medicine, only a few qualitative studies exploring AM prescribing behaviour of veterinarians have been performed, primarily in production animals (Mateus et al., 2014; Speksnijder et al., 2015a; Coyne et al., 2016; Currie et al., 2018). As part of a larger research project on antimicrobial stewardship in Dutch companion animal clinics (the ASAP project: Antimicrobial Stewardship and Pets), the present study aimed to identify factors influencing AM prescribing in companion animal clinics in the Netherlands. This information will act as a starting point for the development and implementation of an antimicrobial stewardship programme for companion animal clinics.

\section{Material and methods}

\subsection{Study design}

Face-to-face interviews were used to qualitatively examine the spectrum of factors that might influence AM prescribing behaviour of Dutch companion animal veterinarians. A semi-structured interview guide was developed by the authors ( $\mathrm{NH}, \mathrm{MH}, \mathrm{DS}$ and $\mathrm{EB})$ based upon their field experience as well as the models of Hulscher et al. (2010b) and Flottorp et al. (2013) (Appendix A in Supplementary material). Three pilot interviews were conducted to validate and adjust the guide where necessary. Interviews were started with a general 'warm-up' question about the clinic. Subsequently, participants were asked to describe a recent clinical case in which they prescribed AMs and a case in which they doubted about prescribing AMs. Next, the remaining topics from the interview guide were discussed. The following topics were covered during the interviews (in free order): prior knowledge on use and legislation of antimicrobials; opinions and behaviour of the veterinarian influencing AMU; cultural and socioeconomic context; behaviour of the companion animal owner and organisation of care within the veterinary clinic (Appendix A in Supplementary material). Participants were encouraged to reflect on their opinion in detail and to discuss the topics freely.

Interviews were held between January and April 2015 by NH (researcher and DVM). Interviews were recorded and subsequently transcribed verbatim by an independent transcription company. Interviews were analysed as soon as possible, enabling an iterative process with minor changes of the interview guide based upon emerging themes and potential missing information.

\subsection{Selection of participants}

An exhaustive list of all Dutch veterinarians (from the Royal Netherlands Veterinary Association, KNMvD) was used for the selection of companion animal veterinarians. Inclusion criteria were: practising veterinarians with a primary focus on companion animals (i.e. dogs, cats, rabbits and other small pets, including birds and exotics).
Selection of veterinarians was done purposefully to ensure variation in gender, age, years of professional experience, clinical setting (e.g. solo versus group practice and referral versus first opinion clinics) and geographical location.

Interviews were held, and veterinarians were invited on an ongoing basis, until theoretical data saturation was reached, i.e. no new information could be identified from the last three consecutive interviews. Veterinarians were invited via e-mail first, followed by a phone call to answer questions and to arrange a time and place for the interview. Interviews took place at a convenient location for the veterinarian, which could be at their clinic or home or at the researcher's workplace. Before enrolment, all veterinarians received written information describing the purpose of the interview and the voluntary nature of participation. All interviewed veterinarians signed an informed consent to assure confidential handling of the data and permission to record the interviews. Interviewed veterinarians were financially compensated for their time investment. This study was exempt from ethical approval according to Dutch legislation (Law on Medical Scientific Research with People) because no patients were involved.

\subsection{Data analysis}

ATLAS.ti 7.5 (ATLAS.ti Scientific Software Development GmbH, Berlin, Germany) was used to manage and analyse the qualitative data. An iterative approach was used to analyse interview notes and interview transcripts on an ongoing basis. Two questions were leading during the analysis of the data 'which factors influence the decision to prescribe antimicrobials' and 'which factors influence which antimicrobial to prescribe'. Interviews were conducted in Dutch and analyses of the transcripts were also performed in Dutch. A native English speaker translated quotations used in the manuscript.

All interview transcripts were coded and analysed by $\mathrm{NH}$ in a stepwise fashion as prescribed by van Buul et al. (2014). Eight randomly selected transcripts were independently analysed and coded by two other researchers (HG and EB, four transcripts each). The doubleanalysed transcripts were used for validation purposes and to discuss the coding process. During the coding and analysis of the remaining transcripts (by $\mathrm{NH}$ ) several meetings (with HG and $\mathrm{EB}$ ) took place to discuss the transcripts and coding process until consensus was reached. Emerging themes and patterns were discussed as well.

After the analysis of all transcripts, factors influencing AM prescribing behaviour were discussed with HG, EB and MH until consensus was reached. Finally, a draft conceptual model presenting these factors was developed. This model and observed patterns were critically evaluated (15 October 2015) by the ASAP project group, which consisted of stakeholders from veterinary and human medicine. Finally, the model was revised during the writing process.

The consolidated criteria for reporting qualitative research (COREQ) and the standards for reporting Qualitative Research (SRQR) were used as checklists (Tong et al., 2007; O'Brien et al., 2014) to guide a comprehensive reporting of the study.

\section{Results}

\subsection{Demographic data}

Theoretical data saturation was reached after 18 interviews. This number of interviews was reached by inviting 25 companion animal veterinarians; 7 veterinarians were not willing to participate, mainly due to lack of time or interest. Duration of the interviews varied from 50 to $90 \mathrm{~min}$. The interviewed veterinarians worked in different clinics and settings, spread throughout the Netherlands. One veterinary European Board-Certified specialist was included, one veterinarian mainly working in shelters, one veterinarian working in a holistic clinic and two veterinarians working in a corporate chain of veterinary 
Table 1

Demographics of interviewed companion animal veterinarians $(n=18)$.

\begin{tabular}{lll}
\hline Demographic & & Veterinarians (n $=18)$ \\
\hline Gender & Male & 5 \\
& Female & 13 \\
\hline \multirow{2}{*}{ Years since graduation } & Mean (range) & $18.2(2-38)$ yrs \\
& $<10$ yrs & 5 \\
& $10-20$ yrs & 6 \\
& $21-30$ yrs & 4 \\
& $>30$ yrs & 3 \\
\hline \multirow{2}{*}{ Country of graduation } & The Netherlands & 16 \\
& Belgium & 2 \\
\hline \multirow{2}{*}{ Age } & Mean (range) & $45.9(26-68)$ yrs \\
& $<30$ yrs & 2 \\
& $30-40$ yrs & 3 \\
& $40-50$ yrs & 5 \\
& $>50$ yrs & 8 \\
\hline \multirow{2}{*}{ Position } & Employer/practice owner & 10 \\
& employee &
\end{tabular}

clinics. Except for one, all veterinarians treated companion animals only. Table 1 shows additional demographic information of the interviewed veterinarians.

\subsection{Factors influencing antimicrobial prescribing behaviour}

In the conceptual model, factors influencing AM prescribing behaviour were divided into four major categories: 1) veterinarian-related factors, 2) patient-related factors (owner- and pet-related factors), 3) treatment-related factors (i.e. alternative treatment options and antimicrobial-related factors) and 4) contextual factors (i.e. professional interactions, further diagnostics and environmental factors). Fig. 1 shows the conceptual model of factors that influence the AM prescribing behaviour of Dutch companion animal veterinarians. It shows the relation between intrinsic (veterinarian related) and external factors.

\subsubsection{Veterinarian-related factors}

By law, a veterinarian is the authorised person for prescribing AMs for veterinary use. All prescriptions of AMs for veterinary use are therefore made by a veterinarian, which is why 'veterinarian-related factors' is centred in Fig. 1. Interviews showed several intrinsic factors related to the companion animal veterinarian that influence AM prescribing behaviour.

3.2.1.1. Personal beliefs on AMU, opinions, preferences, aversions, experiences and habit. Personal beliefs, opinions and preferences influenced the decision whether to prescribe an AM or not. Personal preferences were regularly stated as a reason to choose a specific AM: "never change a winning team, as then everything always works out" (P8). These preferences could be in line with theoretical knowledge, evidence-based veterinary medicine (EBVM) and current guidelines, but they could also be conflicting. Previous experiences (positive and negative) with prescribing or non-prescribing of AMs appeared to contribute to current decisions, regardless of argumentation based on theory and EBVM. Some veterinarians mentioned prescribing AMs in situations where other veterinarians would seldom prescribe, e.g. perioperatively in elective surgery. Some veterinarians never prescribed AMs during or after elective surgery: "in my view, if you always work meticulously and have a clean operating theatre, then there is no need to prescribe AMs" (P18), whereas others used AMs routinely. Regarding the type of AM chosen, several veterinarians stated mainly choosing AMs according to habit and not so much on the basis of EBVM or guidelines. Besides differences in AMU habits, there were remarkable differences in opinions on the importance of AMR. A considerable number of interviewed veterinarians recognised the need for a more prudent AMU, not only in large animal practice but also in companion animal practice: "it is nothing short of logical that we have become reserved in the use of AMs. Partly because .... Hardly any new AMs are being developed [....] So that is the responsibility that we have" (P12). However, other veterinarians questioned the contribution of AMU in companion animals to problems with AMR seen in human medicine. These veterinarians pointed at AMU in large animals and/or in human medicine as main contributors: "I think that every bit helps, but I think the bits from pets are very small. However, if you were talking about farm animals and would say 'you cannot simply use that anymore', then I could understand your perspective because they administer such vast amounts. But in the case of just $0.3 c c$ for a cat, I would think 'oh, well'......" (P8). A minority of veterinarians questioned the effectiveness of prudent AMU in Dutch companion animals, as long as in other countries AMs are widely available even without prescription. Some veterinarians admitted ignoring current guidelines for companion animals occasionally, because of lack of a real 'problem recognition'.

3.2.1.2. Personal 'guideline' beliefs, opinions, preferences, aversions, experiences and habit. Under Dutch law, the use of fluoroquinolones and $3^{\text {rd }}$ and $4^{\text {th }}$ generation cephalosporins (i.e. third choice AMs) in animals is restricted to infections caused by bacteria with susceptibility results indicating no susceptibility to any other AM. Besides legislation, there are several Dutch guidelines on AMU in companion animals (www.wvab.knmvd.nl). Some veterinarians used the Dutch guidelines to promote prudent AMU as a reminder or back-up. A younger veterinarian stated: "I think that especially when you have just started, you have to deal with so many different things that you simply cannot

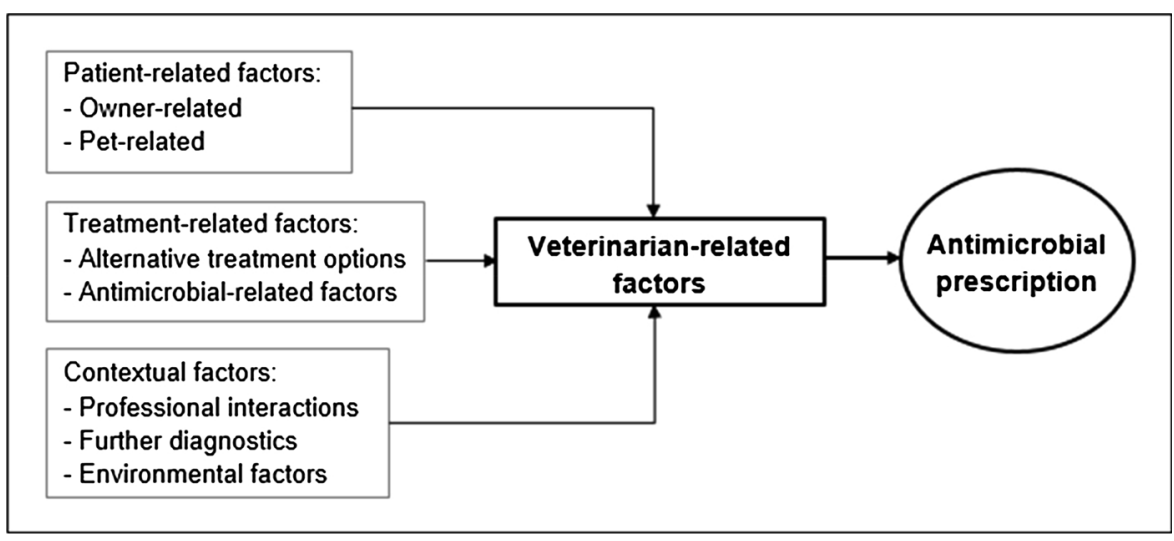

Fig. 1. Conceptual model of factors influencing antimicrobial prescribing behaviour. 
remember everything" (P9). Some used the guidelines as a pressure tool to convince an owner that AMs were not indicated or that further diagnostics had to be performed first. "Yes, I'm sorry, the legislation has changed, I can't give you anything. Just turn on your TV and you'll see the criticism we are facing as vets. And there are good reasons for that, I'm... simply not allowed to" (P13). Not all veterinarians were familiar with the guidelines, while others indicated that the guidelines were unclear or impractical. Some veterinarians indicated being afraid of reprisal when prescribing fluoroquinolones or $3^{\text {rd }}$ or $4^{\text {th }}$ generation cephalosporins: "and to be honest, I hardly dare to use it anymore, in fact only after a positive susceptibility test" (P3), whereas others indicated to use these AMs on a regular basis, even without meeting the legal requirements for use.

3.2.1.3. Veterinarians' knowledge of infectious diseases, antimicrobials and pharmacokinetics. In some cases, theoretical knowledge of infectious diseases and antimicrobials was explicitly mentioned as an influencing factor. Especially when choosing a specific AM, knowledge of the spectrum of activity and sometimes the pharmacokinetics was applied. Veterinary literature and continuing education were also mentioned as influencing factors. However, in some cases, the content of courses or (international) literature conflicted with Dutch legislation.

3.2.1.4. Perceived risks of (non-) treatment and perceived risks of treatment with specific antimicrobials. Fear of complications or an unsatisfied owner after not prescribing AMs was often mentioned as a reason to prescribe: "yes, I think it is because it has become a habit and because one is afraid to leave it out in case it would then go wrong" (P10). Some veterinarians prescribed AMs post-surgery because of a fear of complications due to a lack of hygiene during surgery: "then just to be sure, I will prescribe AM tablets for the cat, because I cannot guarantee that I have worked meticulously and sterilely enough. And that is not a risk I am willing to take" (P4). On the other hand, some veterinarians mentioned fear to prescribe AMs because of harmful side effects: "as otherwise those AMs might cause more harm than the problem they will solve" (P1). Some veterinarians clearly expressed their fear of adverse clinical side effects related to specific AMs. This fear could be based on personal experiences, but also on 'hearsay'. Trimethoprim/sulphonamide combinations were often mentioned in this context: "in such a case, I am so worried about a keratitis sicca that I then deliberately do not do it" (P15).

3.2.1.5. 'Just give it a try' and 'quick fix'. In some cases, veterinarians were uncertain (about a diagnosis and/or which AM to choose) and decided to "give it a try", without a very strong reason or argument. In these cases "It does not hurt to try" was sometimes the driving factor for prescribing AMs. In other cases, a 'quick fix' (for the veterinarian and/ or owner) was stated as a driving factor.

\subsubsection{Patient-related factors}

In veterinary medicine, a pet is almost always accompanied by an owner or caretaker. In the conceptual model, both the pet and owner were identified as influencing factors.

\subsubsection{Pet-related factors}

3.2.2.1.1. Pet characteristics. Interviews indicated that AMU was influenced by pet characteristics, e.g. animal species, breed, age, living conditions, weight, coat type and character of the pet. In the case of a non-cooperative or aggressive animal, veterinarians tended to more frequently choose a therapy with AMs, even when AMs were not necessarily indicated. This was mainly because a physical and/or further examination was not feasible and because alternative treatment options were not applicable. In the case of an aggressive animal, veterinarians seemed to prefer injectable AMs and long-acting AMs. Living conditions were also mentioned as an influencing factor, e.g. a household with young children for which the veterinarian was concerned about the possible spread of bacteria to the children and therefore preferred AMs above alternative treatment options. In the case of a large or heavy animal, veterinarians sometimes chose another type of AM to keep down the costs or the number of tablets that had to be administered to the animal.

3.2.2.1.2. Current clinical situation and history. The current clinical situation, as well as the clinical history, influenced the prescribing decision. The interviews showed that AMs were predominantly prescribed on the basis of presumed diagnoses: "more often than not, you do not have a $100 \%$ diagnosis but instead a probable diagnosis, and that is what you act on" (P12). 'Only mild' signs were regularly stated as a reason not to prescribe AMs, 'severe' clinical signs and especially pyrexia were mentioned as a reason to prescribe; " $a$ fever of 41 degrees Celsius, was severely ill" (P1). Comorbidities and previous episodes of similar symptoms were also mentioned as influencing factors. The localisation of an injury or type of surgery, organ(s) involved, possible causal agents and the severity of complaints also influenced the AM chosen: "what kind of inflammation it is, where it is located, the severity.... and uhm.... Possible causal agents you might think of..." (P4).

\subsubsection{Owner-related factors}

3.2.2.2.1. Owner demands. In several cases, veterinarians mentioned perceived pressure from owners to prescribe AMs. Some owners were very clear in their request: "they simply say in no uncertain terms: I want AMs, full stop. You may only look further once we have tried this because I am convinced it works simply because it has worked in the past" (P13). In other cases, the pressure was less clear: "sometimes I do think: I'll just prescribe [an AM] as otherwise they will simply not be satisfied, even though it would be better for the dog not to and it is not necessary either" (P4). Some veterinarians also mentioned perceiving pressure not to prescribe AMs: "that they just don't want [to use AMs] at all, because they do not want to vaccinate either" (P16). Breeders were specifically mentioned as quite demanding regarding the prescribing of AMs: "some breeders who are very demanding and who even think that they know when they need AMs" (P14).

3.2.2.2.2. Owner convenience and costs. The choice for a specific AM could also be influenced by the owner. Convenience for an owner and expected compliance of a proposed therapy were explicitly mentioned as influencing factors. Costs of and the willingness to pay for treatment were also influential. In some cases, owners refused or did not want to pay for any further diagnostics limiting the veterinarians' options to make a proper diagnosis. In some cases, when owners had a pet insurance, additional diagnostics were performed more easily or a more expensive AM was given. The familiarity with an owner and the veterinarian's assessment of the capacity of an owner or caretaker to take care of an animal and to notice deterioration on time were also mentioned as influencing factors: "I know them quite well, and then I think... well.... If you don't trust it, just come and see me immediately, but in this case, I thought, if it goes wrong, they will not even notice it..." (P5).

\subsubsection{Treatment-related factors}

3.2.3.1. Alternative treatment options. The interviews showed that alternative treatment options, defined here as 'all non-antimicrobial treatment options' could influence the decision 'to prescribe AMs'. In several cases, veterinarians appeared to be willing to try nonantimicrobial (supportive) treatment options, but when an alternative option 'appeared' ineffective (based upon earlier experience and expectation) or when an owner had to be convinced to apply an alternative option, veterinarians tended to choose for AMs. The application of alternative treatment options had to be feasible for an owner, for example when using local skin shampoos instead of systemic AMs in case of dermatologic problems. The use of alternative treatment options also depended on the experience with these options and preference of the veterinarian. Some combination products containing AMs were perceived to be more user friendly and/or cheaper compared 
to products not containing AMs, e.g. local skin or ear products with AMs and steroids: "on the face of it, those AMs in that skin product are often not even necessary. However, the one without AMs is a spray and the one with AMs is an ointment, and there is a difference in costs as well. I believe the spray is at least twice as expensive" (P17).

3.2.3.2. Antimicrobial-related factors. Antimicrobial- and formulationrelated factors appeared to have an important influence on deciding 'which antimicrobial to prescribe'. Practical considerations, side effects, spectrum of action, AM classification (first, second or third choice according to Dutch policy on AMU), route of administration, duration of action, immune-modulating effects, instructions for use, expiration date, package size and costs of specific products containing AMs were mentioned as influencing factors. Practical considerations were often mentioned as motivating factors. Reasons from one veterinarian to choose amoxicillin in a specific situation: "because it is cheap, easy to dose, is a fine second choice AM, I can inject it..." (P2). Taste and acceptance by the animal were also mentioned as reasons to choose for a specific AM. Other practical considerations were also mentioned: "I really look at what I think is a nice product, what I think is a suitable packaging, what I find easy to use and what I think from my impressions of the owner will be good and easy for them to administer" (P18). The duration of action was often mentioned as a driver to choose for a specific AM. When choosing a long-acting injectable AM, the AM class seemed to be of secondary importance compared to the duration of action and the route of administration. One specific long-acting injectable preparation containing cefovecin was regularly chosen because of the convenience (for veterinarian and owner), the compliance and as a solution for non-cooperative animals. However, some veterinarians had an aversion to long-acting AMs: "but that injection works for $48 \mathrm{~h}$, which I am not entirely happy with anyway, because it is such a slow release AM that only releases a bit of AM once in a while" (P7).

\subsubsection{Contextual factors}

In the conceptual model, several contextual factors influencing AM prescribing were identified. These factors were mainly linked to the workplace and colleagues at work.

3.2.4.1. Professional interactions. Veterinarians mentioned influence from direct colleagues on AM prescribing. Some clinics had strong habits and (written) policies regarding AMU, whereas in other clinics these were absent. In some clinics, veterinarians perceived pressure from other colleagues to prescribe AMs: "look, I am always happy to talk about the matter, but it remains his word. Nevertheless, to put things bluntly, I must do what he says if I want to keep my job" (P7). On the other hand, younger and/or recently graduated veterinarians were reported to have an influence on older veterinarians as well: "every so often, when a new colleague comes, such as $\mathrm{XXX}$, then they might suggest 'why don't you try this?' And then I think to myself, that is another viable option, of course" (P3). Some veterinarians indicated that newly graduated veterinarians tended to be more prudent in AM prescribing compared to older veterinarians: "I have to be honest that the younger veterinarians are quite strict, but that we also have some older colleagues who somewhat more easily say, well.... let's just do it. And that is a bit of a discussion here" (P14). Not only differences between colleagues within the same clinic, but also differences in AM policies between clinics were mentioned: "and then they leave and go to another veterinarian where they can get the injection, and that's fine with me" (P2). Even though different policies between clinics were explicitly mentioned, most veterinarians indicated that they adhered to their own policies: "and then you have a few who say: then I'll just go and get it somewhere else. And then it is the responsibility of the veterinarian who does that" (P12). Veterinary specialists and human doctors were also mentioned as factors influencing AM prescribing behaviour. In some cases, however, the advice of specialists appeared to conflict with Dutch legislation.
3.2.4.2. Further diagnostics. In addition to bacterial culture and sensitivity testing, blood work, radiographs, urinalysis, cytology (mainly skin or ear samples), ultrasound and other forms of further diagnostics were mentioned as influencing the prescribing decision: " $I$ believe that the rule is that you must diagnose first; there must be a clear evidence-based diagnosis and, whenever possible, you have to test susceptibility or take a first choice AM" (P12) and "if, for example, there was a lot of blood, leucocytes and proteins on my urinary stick, then I would prescribe AMs" (P9).

\subsubsection{Environmental factors}

3.2.4.3.1. Time. In some cases, time was a limiting factor for performing further diagnostics and/or evaluating a patient thoroughly. In these cases, prescribing AMs appeared to be a quick solution. Time needed to obtain laboratory results, especially in the case of bacterial culture and sensitivity testing, was regularly mentioned as a limiting factor for performing further diagnostics.

3.2.4.3.2. Availability. A specific type of AM can only be prescribed if it is available, both in the clinic as well as from the wholesalers. In some cases, for example, only amoxicillin with clavulanic acid was available in the clinic. As a result, veterinarians were not able to prescribe amoxicillin without clavulanic acid even if this was preferred. Another regularly mentioned example was the limited availability of topical formulations with corticosteroids but without AMs. When a specific AM was not in stock, it could not be prescribed, even when an owner specifically asked for it. Here it could serve as an argument to convince an owner to try another type of AM or an alternative treatment option.

3.2.4.3.3. Commercial drivers. A very small number of veterinarians stated that financial gain could influence AM prescribing. In the case of shelter medicine, it was recognised that AMs were sometimes prescribed to decrease the number of days that a dog or cat had to stay in isolation and with that costs for the shelter. Only a few veterinarians mentioned that violating regulations and guidelines (e.g. prescribing fluoroquinolones without susceptibility testing) could attract new clients: "yes, and it can also get me clients sometimes" (P5).

\section{Discussion}

This study explored the spectrum of factors influencing Dutch companion animal veterinarians in the decision-making process on prescribing AMs. In the conceptual model, four major categories of influencing factors could be identified: 1) veterinarian-related factors, 2) patient-related factors, 3) treatment-related factors and 4) contextual factors. Many of the factors described are related, but ultimately the companion animal veterinarian is authorised to prescribe AMs.

The decision-making process of companion animal veterinarians on AM prescribing appeared to be complex, which is recognised both in human and veterinary medicine (Hulscher et al., 2010b; van Buul et al., 2014; Speksnijder et al., 2015a; Coyne et al., 2016). Many factors other than theoretical knowledge and clinical reasoning appeared to play a role. Other qualitative studies on AM prescribing behaviour have also shown this (Coenen et al., 2000; Hulscher et al., 2010b; Fleming et al., 2014; Mateus et al., 2014; Currie et al., 2018). As the person authorised to prescribe AMs, the companion animal veterinarian appeared to play a central role in the decision-making process.

Over the past few decades, numerous clinical practice guidelines have been developed and implemented in human medicine (e.g. www.escmid.org). These comprehensive guidelines are usually based on substantial amounts of scientific evidence. In veterinary medicine, guidelines are less well established, and for several infections the level of scientific evidence on optimal AM treatment strategies is limited (Jessen et al., 2015). Dutch legislation on veterinary AMU was revised in the period 2012-2013 and guidelines on prudent AMU have been developed. This study showed that the familiarity with and implementation of these guidelines for a prudent AMU in companion 
animals differed considerably among the veterinarians interviewed. Other studies have also confirmed this finding (Jessen et al., 2017; Van Cleven et al., 2018). Although our study was not designed for a quantitative exploration of influencing factors, newly graduated companion animal veterinarians seemed to be more familiar with the guidelines compared to more experienced veterinarians. This is possibly because policies and guidelines on AMU are now embedded in education. Guidelines seem to support younger veterinarians in daily practice, whereas more experienced veterinarians seem to be less willing to adjust their daily routines (Speksnijder et al., 2015b). Studies are needed to measure and improve implementation of and compliance to current guidelines. Guidelines should also be evaluated and updated regularly taking results from qualitative studies into account. For example, in our study (perceived) risks of antimicrobial treatment or the opposite (perceived) risks of no antimicrobial treatment were mentioned quite often.

Opinions on the importance of AMR and prudent AMU in companion animals appeared to differ considerably between the veterinarians interviewed. These differences in opinions were also observed among Dutch farm animal veterinarians (Speksnijder et al., 2015b). Evidencebased information on the importance of prudent AMU in companion animals and improved awareness are needed to optimise AMU.

Patient-related factors appeared to be important as well, which is in line with other studies confirming the differences in type of AM chosen and the route of administration between dogs and cats (Buckland et al., 2016; Singleton et al., 2017). Especially in the case of the use of cefovecin in cats, not so much the AM spectrum, but the character of the animal and the product characteristics (injection and long-acting) appeared to be reasons to prescribe this specific AM. Other studies have shown this as well (Burke et al., 2017; Hardefeldt et al., 2017). Animal characteristics are probably difficult to change. However, future research should be based on finding solutions for treating non-cooperative animals in line with prudent AMU principles.

The importance of an animal owner or caretaker regarding AMU is confirmed by other studies on companion animals as well as on farm animals (Mateus et al., 2014; Speksnijder et al., 2015a; Coyne et al., 2016; Chipangura et al., 2017; Hardefeldt et al., 2017; Smith et al., 2018). A recent study on AM prescribing in companion animals states that veterinarian's perceptions of client expectations might drive inappropriate AM prescribing (Currie et al., 2018). In human medicine, the patient or his/her family can influence the decision-making process as well (van Buul et al., 2014; Halls et al., 2017; Bosley et al., 2018). These findings show that educating owners, involving owners in prudent AMU and a better explanation of restricted AMU are needed to optimise AMU. International and national veterinary associations have already developed educational posters, but the distribution, practical use and effect of these documents have not been evaluated yet (e.g. FECAVA, FVE and KNMvD).

The unwillingness or inability to pay for further diagnostics or therapy was identified as a factor that may lead to inappropriate AMU. In human medicine, especially non-hospital settings, a lack of diagnostic resources was also mentioned as a factor that contributes to diagnostic uncertainty (van Buul et al., 2014; Anthierens et al., 2015). Further diagnostics are needed to reduce diagnostic uncertainty. In contrast to human medicine, most Dutch companion animal owners do not have a pet insurance, and so the owner has to pay the costs of diagnostics and therapy. Financial barriers play an important role in treatment decisions of veterinarians, as was also shown in previous research (Mateus et al., 2014; Hardefeldt et al., 2017). The development of cheaper, faster and more reliable on-site diagnostics might lead to better adherence to current guidelines and a decrease in inappropriate AMU.

Treatment-related factors, especially practical considerations like administration route, lack of adverse effects, and availability turned out to play another important role. Pharmaceutical companies can take more responsibility here, e.g. by producing easy-to-administer first choice AMs (e.g. tasty liquid oral formulations instead of tablets) or smaller packages (to prevent expiration of less often used AMs) and by marketing alternative (non-antimicrobial) treatment options. Registration of new veterinary medicinal products is time-consuming and expensive, but smaller packages of tablets, for example, might be easier to accomplish.

Many contextual factors, especially peer pressure, appeared to influence a companion animal veterinarian. A clear and uniform clinic policy on prudent AMU might increase awareness and decrease differences in treatment regimens between colleagues within a clinic.

This study has various strengths and limitations. Individual interviews were preferred over focus groups to ensure unbiased and truthful answers from participants (Sanchez et al., 2014). During the interview process, answers appeared to be very open and without scruples, which reassured the authors that the answers were honest and unbiased. Veterinarians from different settings, supporting and opposing current policies on AMU, were interviewed. We are therefore confident that we have covered the broad array of influencing factors. Relatively more female veterinarians than male veterinarians were interviewed reflecting the current situation in Dutch companion animal veterinarians.

Because a qualitative strategy was used, it was not possible to quantify the importance of the different influencing factors (Pope et al., 2002). However, this qualitative study generated a broad set of factors influencing Dutch companion animal veterinarians and an in-depth understanding of these factors, which was the purpose of this study. Because legislation and organisation of veterinary care varies between countries, some factors might be restricted to the Dutch situation, other factors might be more generalised. Few qualitative studies have been done on this topic in companion animal medicine, but many factors seem to be similar to factors identified in human medicine and farm animal medicine in different countries.

Findings from the current study will be quantitatively further explored and used to develop tailored interventions to promote responsible AMU in Dutch companion animal clinics within an antimicrobial stewardship programme (ASP). Antimicrobial stewardship is usually defined as a multifaceted and dynamic approach to preserve the clinical efficacy of AMs by optimising AMU while minimising the emergence of AMR and possible other adverse effects (Guardabassi and Prescott, 2015; Prescott and Boerlin, 2016). When developing an ASP, the primary focus should be on the companion animal veterinarian. Improving awareness of the importance of prudent AMU and AMU guidelines seems to be a major issue that needs to be taken into account. Companion animal owners should be involved more and practical solutions need to be found to support veterinarians in responsible AMU, also in non-cooperative animals. Reducing barriers for performing further diagnostics might also contribute to a more prudent AMU.

\section{Funding}

This study was funded by ZonMw (Netherlands Organisation for Health Research and Development, The Hague, the Netherlands), project number 205300003.

\section{Submission declaration}

This research study has not been previously published elsewhere.

\section{Conflicts of interest}

This study was funded by ZonMw (Netherlands Organisation for Health Research and Development, The Hague, the Netherlands), project number 205300003.

\section{Acknowledgements}

We are very grateful to the participating veterinarians for their open 
and honest cooperation. We also thank the remaining members of the ASAP project group (J.T. Bosje, I.M. van Geijlswijk, D. Heederik, L.J. Hellebrekers, M.F.M. Langelaar, J.M. Prins, L. Schipper and J.M. Verheij) for their input.

\section{Appendix A. Supplementary data}

Supplementary material related to this article can be found, in the online version, at doi:https://doi.org/10.1016/j.prevetmed.2018.07. 013.

\section{References}

AMCRA (Antimicrobial Consumption and Resistance in Animals), www.amcra.be/nl/ formularia/formularia, last Accessed 15 February 2018.

Anthierens, S., Tonkin-Crine, S., Cals, J.W., Coenen, S., Yardley, L., Brookes-Howell, L., Fernandez-Vandellos, P., Krawczyk, J., Godycki-Cwirko, M., Llor, C., Butler, C.C., Verheij, T., Goossens, H., Little, P., Francis, N.A., 2015. Clinicians' views and experiences of interventions to enhance the quality of antibiotic prescribing for acute respiratory tract infections. J. Gen. Intern. Med. 30, 408-416.

Baede, V.O., Wagenaar, J.A., Broens, E.M., Duim, B., Dohmen, W., Nijsse, R., Timmerman, A.J., Hordijk, J., 2015. Longitudinal study of extended-spectrum-betalactamase- and AmpC-producing enterobacteriaceae in household dogs. Antimicrob. Agents Chemother. 59, 3117-3124.

Bosley, H., Henshall, C., Appleton, J.V., Jackson, D., 2018. A systematic review to explore influences on parental attitudes towards antibiotic prescribing in children. J. Clin. Nurs. 27 (5-6), 892-905.

BSAVA (British Small Animal Veterinary Association), www.bsava.com/Resources/ Veterinary-resources/Medicines-Guide/Antibacterials, last Accessed 15 February 2018.

Buckland, E.L., O'Neill, D., Summers, J., Mateus, A., Church, D., Redmond, L., Brodbelt, D., 2016. Characterisation of antimicrobial usage in cats and dogs attending UK primary care companion animal veterinary practices. Vet. Rec. 179, 489.

Burke, S., Black, V., Sánchez-Vizcaíno, F., Radford, A., Hibbert, A., Tasker, S., 2017. Use of cefovecin in a UK population of cats attending first-opinion practices as recorded in electronic health records. J. Feline Med. Surg. 19, 687-692.

Chipangura, J.K., Eagar, H., Kgoete, M., Abernethy, D., Naidoo, V., 2017. An investigation of antimicrobial usage patterns by small animal veterinarians in South Africa. Prev. Vet. Med. 136, 29-38.

Chomel, B., Sun, B., 2011. Zoonoses in the bedroom. Emerg. Infect. Dis. 17, 167-172.

Coenen, S., Van Royen, P., Vermeire, E., Hermann, I., Denekens, J., 2000. Antibiotics for coughing in general practice: a qualitative decision analysis. Fam. Pract. 17, 380-385.

Collignon, P.C., Conly, J.M., Andremont, A., McEwen, S.A., Aidara-Kane, A., World Health Organization Advisory Group, Bogota Meeting on Integrated Surveillance of Antimicrobial Resistance (WHO-AGISAR), Agerso, Y., Andremont, A., Collignon, P., Conly, J., Dang Ninh, T., Donado-Godoy, P., Fedorka-Cray, P., Fernandez, H., Galas, M., Irwin, R., Karp, B., Matar, G., McDermott, P., McEwen, S., Mitema, E., ReidSmith, R., Scott, H.M., Singh, R., DeWaal, C.S., Stelling, J., Toleman, M., Watanabe, H., Woo, G.J., 2016. World health organization ranking of antimicrobials according to their importance in human medicine: a critical step for developing risk management strategies to control antimicrobial resistance from food animal production. Clin. Infect. Dis. 63, 1087-1093.

Coyne, L.A., Latham, S.M., Williams, N.J., Dawson, S., Donald, I.J., Pearson, R.B., Smith, R.F., Pinchbeck, G.L., 2016. Understanding the culture of antimicrobial prescribing in agriculture: a qualitative study of UK pig veterinary surgeons. J. Antimicrob. Chemother. 71, 3300-3312.

Currie, K., King, C., Nuttall, T., Smith, M., Flowers, P., 2018. Expert consensus regarding drivers of antimicrobial stewardship in companion animal veterinary practice: a Delphi study. Vet. Rec. 182, 691

Duim, B., Verstappen, K.M., Broens, E.M., Laarhoven, L.M., van Duijkeren, E., Hordijk, J., de Heus, P., Spaninks, M., Timmerman, A.J., Wagenaar, J.A., 2016. Changes in the population of Methicillin-Resistant Staphylococcus pseudintermedius and dissemination of antimicrobial-resistant phenotypes in the Netherlands. J. Clin. Microbiol. 54, 283-288.

Dyar, O.J., Huttner, B., Schouten, J., Pulcini, C., ESGAP, 2017. What is antimicrobial stewardship? Clin. Microbiol. Infect. 23 (11), 793-798.

EMA (European Medicines Agency), 2015. Reflection Paper on the Risk of Antimicrobial Resistance Transfer From Companion Animals. http://www.ema.europa.eu/docs/ en_GB/document_library/Scientific_guideline/2015/01/WC500181642.pdf.

ESCMID (European Society of Clinical Microbiology and Infectious Diseases), https:// www.escmid.org/escmid publications/medical guidelines/escmid guidelines/, last Accessed 10 January 2018.

FECAVA (Federation of Companion Animal Veterinary Associations), FECAVA Advice to Companion Animal Owners on Responsible Use of Antibiotics and Infection control, http://www.fecava.org/sites/default/files/files/2014_12_fecava_advise\%20owners. pdf, last Accessed 10 January 2018.

Fleming, A., Bradley, C., Cullinan, S., Byrne, S., 2014. Antibiotic prescribing in long-term care facilities: a qualitative, multidisciplinary investigation. BMJ Open 4 e0064422014-006442.

Flottorp, S., Oxman, A., Krause, J., Musila, N., Wensing, M., Godycki Cwirko, M., Baker, R., Eccles, M., 2013. A checklist for identifying determinants of practice: A systematic review and synthesis of frameworks and taxonomies of factors that prevent or enable improvements in healthcare professional practice. Implement. Sci. 8, 35.

FVE (Federation of Veterinarians of Europe), Veterinarians care for animals and people, http://www.fve.org/uploads/publications/docs/fve_antimicrobials_pets_final_small. pdf, last Accessed 10 January 2018.

Guardabassi, L., Prescott, J.F., 2015. Antimicrobial stewardship in small animal veterinary practice: from theory to practice. Vet. Clin. North Am. Small Anim. Pract. 45, 361-376 vii.

Guardabassi, L., Schwarz, S., Lloyd, D.H., 2004. Pet animals as reservoirs of antimicrobial-resistant bacteria. J. Antimicrob. Chemother. 54, 321-332.

Halls, A., Van’t Hoff, C., Little, P., Verheij, T., Leydon, G.M., 2017. Qualitative interview study of parents' perspectives, concerns and experiences of the management of lower respiratory tract infections in children in primary care. BMJ Open 7 e015701-2016015701.

Hardefeldt, L.Y., Holloway, S., Trott, D.J., Shipstone, M., Barrs, V.R., Malik, R., Burrows, M., Armstrong, S., Browning, G.F., Stevenson, M., 2017. Antimicrobial prescribing in dogs and cats in Australia: results of the Australasian infectious disease advisory panel survey. J. Vet. Intern. Med. 31, 1100-1107.

Hulscher, M.E.J.L., Grol, R.P.T.M., van der Meer, J.W.M., 2010a. Antibiotic prescribing in hospitals: A social and behavioural scientific approach. Lancet Infect. Dis. 10, $167-175$.

Hulscher, M.E.J.L., van der Meer, J.W.M., Grol, R.P.T.M., 2010b. Antibiotic use: how to improve it? Int. J. Med. Microbiol. 300, 351-356.

ISCAID (International Society for Companion Animal Infectious Diseases), https://iscaid. org/guidelines\#Antimicrobial\%20use, last Accessed 18 June 2018.

Jessen, L.R., Damborg, P.P., Spohr, A., Schjøth, B., Wiinberg, B., Houser, G., Willesen, J., Schjærff, M., Eriksen, T., Jensen, V.F., Guardabassi, L., 2012. Antibiotic Use Guidelines for Companion Animal Practice. DSAVA. https://www.ddd.dk/sektioner/ familiedyr/antibiotikavejledning/Documents/AntibioticGuidelines\%20-\%20v1.4 jun15.pdf.

Jessen, L.R., Sorensen, T.M., Bjornvad, C.R., Nielsen, S.S., Guardabassi, L., 2015. Effect of antibiotic treatment in canine and feline urinary tract infections: a systematic review. Vet. J. 203, 270-277.

Jessen, L.R., Sorensen, T.M., Lilja, Z.L., Kristensen, M., Hald, T., Damborg, P., 2017. Cross-sectional survey on the use and impact of the Danish national antibiotic use guidelines for companion animal practice. Acta Vet. Scand. 59 81-017-0350-8.

Marques, C., Belas, A., Franco, A., Aboim, C., Gama, L.T., Pomba, C., 2018. Increase in antimicrobial resistance and emergence of major international high-risk clonal lineages in dogs and cats with urinary tract infection: 16 year retrospective study. J. Antimicrob. Chemother. 73, 377-384.

Mateus, A.L.P., Brodbelt, D., Barber, N., Stärk, K.D.C., 2014. Qualitative study of factors associated with antimicrobial usage in seven small animal veterinary practices in the UK. Prev. Vet. Med. 117, 68-78.

Netherlands Veterinary Medicines Institute (SDa), 2017. Usage of Antimicrobial Drugs in Companion Animals 2012-2014; Results of a Survey of Veterinary Practices in the Netherlands. http://www.autoriteitdiergeneesmiddelen.nl/Userfiles/Eng\%20survey $\% 20$ GD\%20en\%20P/engels-def-rapport-survey-ab-gebruik-bij-gezelschapsdierenvoor-website.pdf.

O’Brien, B.C., Harris, I.B., Beckman, T.J., Reed, D.A., Cook, D.A., 2014. Standards for reporting qualitative research: a synthesis of recommendations. Acad. Med. 89, 1245-1251.

Pleydell, E.J., Souphavanh, K., Hill, K.E., French, N.P., Prattley, D.J., 2012. Descriptive epidemiological study of the use of antimicrobial drugs by companion animal veterinarians in New Zealand. N. Z. Vet. J. 60, 115-122.

Pomba, C., Rantala, M., Greko, C., Baptiste, K.E., Catry, B., van Duijkeren, E., Mateus, A. Moreno, M.A., Pyorala, S., Ruzauskas, M., Sanders, P., Teale, C., Threlfall, E.J., Kunsagi, Z., Torren-Edo, J., Jukes, H., Torneke, K., 2017. Public health risk of antimicrobial resistance transfer from companion animals. J. Antimicrob. Chemother. 72, 957-968.

Pope, C., van Royen, P., Baker, R., 2002. Qualitative methods in research on healthcare quality. Qual. Saf. Health Care 11, 148-152.

Prescott, J.F., Boerlin, P., 2016. Antimicrobial use in companion animals and good stewardship practice. Vet. Rec. 179, 486-488.

Sanchez, G., Roberts, R., Albert, A., Johnson, D., Hicks, L., 2014. Effects of knowledge, attitudes, and practices of primary care providers on antibiotic selection, United States. Emerg. Infect. Dis. 20, 2041-2047.

Singleton, D.A., Sanchez-Vizcaino, F., Dawson, S., Jones, P.H., Noble, P.J.M., Pinchbeck, G.L., Williams, N.J., Radford, A.D., 2017. Patterns of antimicrobial agent prescription in a sentinel population of canine and feline veterinary practices in the United Kingdom. Vet. J. 224, 18-24.

Smith, M., King, C., Davis, M., Dickson, A., Park, J., Smith, F., Currie, K., Flowers, P., 2018. Pet owner and vet interactions: exploring the drivers of AMR. Antimicrob. Resist. Infect. Control 7 46-018-0341-1. eCollection 2018.

Speksnijder, D.C., Jaarsma, A.D.C., van der Gugten, A.C., Verheij, T.J.M., Wagenaar, J.A., 2015a. Determinants associated with veterinary antimicrobial prescribing in farm animals in the Netherlands: a qualitative study. Zoonoses Public Health 62 (Suppl. 1), 39-51.

Speksnijder, D.C., Jaarsma, D.A., Verheij, T.J., Wagenaar, J.A., 2015b. Attitudes and perceptions of Dutch veterinarians on their role in the reduction of antimicrobial use in farm animals. Prev. Vet. Med. 121, 365-373.

Teixeira Rodrigues, A., Roque, F., Falcão, A., Figueiras, A., Herdeiro, M., 2013. Understanding physician antibiotic prescribing behaviour: A systematic review of qualitative studies. Int. J. Antimicrob. Agents 41, 203-212.

Tong, A., Sainsbury, P., Craig, J., 2007. Consolidated criteria for reporting qualitative research (COREQ): a 32-item checklist for interviews and focus groups. Int. J. Qual. Health Care 19, 349-357. 
Van Buul, L.W., van der Steen, J.T., Doncker, S.M.M.M., Achterberg, W.P., Schellevis, F.G., Veenhuizen, R.B., Hertogh, C.M.P.M., 2014. Factors influencing antibiotic prescribing in long-term care facilities: a qualitative in-depth study. BMC Geriatr. 14, 136.

Van Cleven, A., Sarrazin, S., de Rooster, H., Paepe, D., Van der Meeren, S., Dewulf, J., 2018. Antimicrobial prescribing behaviour in dogs and cats by Belgian veterinarians. Vet. Rec. 182, 324

WHO, 2015. Global action plan on antimicrobial resistance. last Accessed 5 March 2018. http://www.who.int/antimicrobial-resistance/publications/global-action-plan/en/.
WHO, 2017. Critically Important Antimicrobials for Human Medicine - 5th Review. World Health Organisation 5th Revision 2017.

Worthing, K.A., Abraham, S., Coombs, G.W., Pang, S., Saputra, S., Jordan, D., Trott, D.J., Norris, J.M., 2018. Clonal diversity and geographic distribution of MethicillinResistant Staphylococcus pseudintermedius from Australian animals: Discovery of novel sequence types. Vet. Microbiol. 213, 58-65.

WVAB (Dutch working group on Veterinary Antimicrobial use), www.wvab.knmvd.nl/ formularia, last Accessed 9 April 2018. 